\title{
Evaluation of a novel translational task for assessing emotional biases in different species
}

\author{
Michael H. Anderson • Chloë Hardcastle • \\ Marcus R. Munafò • Emma S. J. Robinson
}

Published online: 20 December 2011

(C) Psychonomic Society, Inc. 2011

\begin{abstract}
Changes in the processing of emotional information are key features of affective disorders. Neuropsychological tests based on emotional faces or words are used to detect emotional/affective biases in humans, but these tests are not applicable to animal species. In the present study, we investigated whether a novel affective tone discrimination task (ATDT), developed to study emotion-related behaviour in rats, could also be used to quantify changes in affective states in humans. To date, the methods used in human neuropsychology have not been applicable to animal experiments. Participants completed a training session in which they learnt to discriminate specific tone frequencies and to correctly respond in order to gain emotionally valenced outcomes, to obtain rewards (money), or to avoid punishment (an aversive sound clip). During a subsequent test session, additional ambiguous probe tones were presented at frequencies intermediate between the reward and avoidance paired tones. At the end of the task, participants completed self-report questionnaires. All participants made more avoidance responses to the most ambiguous tone cues, suggesting a bias towards avoidance of punishment. Individual differences in the degrees of bias observed were correlated with anxiety measures, suggesting the task's sensitivity to differences in state anxiety within a healthy population. Further studies in clinical populations will be necessary to assess the task's sensitivity to pathological anxiety states.
\end{abstract}

M. H. Anderson · C. Hardcastle • E. S. J. Robinson ( $\square)$

School of Physiology and Pharmacology, University of Bristol,

Medical Sciences Building,

Bristol BS8 1TD, United Kingdom

e-mail: pmesjr@bris.ac.uk

M. R. Munafò

School of Experimental Psychology, University of Bristol,

12a Priory Road,

Bristol BS8 1TU, United Kingdom
These data suggest that this affective tone discrimination task provides a novel method to study cognitive affective biases in different species, including humans, and offers a novel assessment to study anxiety.

Keywords Anxiety - Translation · Healthy volunteer . Cognitive affective bias $\cdot$ Emotion

Investigating the neurobiology and treatment of complex psychiatric disorders is often limited by the translational validity of the available animal models. In relation to affective disorders, these issues represent a major hurdle to understanding disease pathologies and to the development of novel drug treatments (Frazer \& Morilak, 2005; McArthur \& Borsini, 2006). Recent studies have suggested that it is feasible to quantify emotion-related behaviours in animals (Enkel et al., 2010; Harding, Paul, \& Mendl, 2004; Paul, Harding, \& Mendl, 2005; Torres et al., 2005). Cognitive processes such as attention, memory, and judgment have been shown to be biased by underlying affective state (Leppänen, 2006). These biases are theorised to alter the interpretation of ambiguous stimuli and, in depression and anxiety, to cause disproportionate encoding and retrieval of negative stimuli (Gotlib, Ranganath, \& Rosenfeld, 1998). Neuropsychological tests of emotional processing almost exclusively utilise stimuli-for instance, facial images or words - that cannot be used with nonhuman animals. On the basis of the same neurocognitive theories that underpin these methods, but working with dimensions that are not species specific, cognitive affective biases have been demonstrated in a number of animal species (Brilot, Asher, \& Bateson, 2010; Brydges, Leach, Nicol, Wright, \& Bateson, 2011; Doyle et al., 2011; Salmeto et al., 2011).

Affective biases have been shown to alter performance to emotionally valenced stimuli in human neuropsychological 
tasks such as the emotional go/no-go, facial recognition, and attention and memory recall paradigms (Bradley, Mogg, \& Millar, 1996; Chamberlain \& Sahakian, 2006; Elliott, Rubinsztein, Sahakian, \& Dolan, 2002; Elliott, Zahn, Deakin, \& Anderson, 2011; Erickson et al., 2005; Leppänen, Milders, Bell, Terriere, \& Hietanen, 2004; Mitterschiffthaler et al., 2008; Mogg, Bradley, Millar, \& White, 1995; Mogg, Bradley, Williams, \& Mathews, 1993; Murphy et al., 1999). Anxiety and depression are often comorbid conditions, however; results from these types of tasks suggest that anxiety is generally associated with biases in attention to threat-related or negative information, whilst depression is associated with memory and interpretation biases (for reviews, see Bar-Haim, Lamy, \& Glickman, 2005; Harmer, Shelley, Cowen, \& Goodwin, 2004; Mathews \& MacLeod, 2005; Mogg \& Bradley, 2002; Wright \& Bower, 1992).

Biases in affective processing in animals have been proposed and are hypothesised to influence how animals interact with their environments (LeDoux, 1995, 1999). Harding, Paul, and Mendl (2004) developed the first method to study cognitive affective bias in animals using a modified emotional go/no-go task in which neutral tones were paired with either a positive (obtain food) or a negative (avoid white noise) reinforcer. Once the association had been learnt, intermediate ambiguous probe tones were presented to the rats and their response bias was quantified. Animals in a putative negative affective state made fewer positive responses and were slower to respond to cues predicting reward, suggesting decreased anticipation of positive events, which may be comparable with findings in human participants with emotional disorders (Wright \& Bower, 1992). Using a similar tone-based task, Enkel et al. (2010) further developed Harding et al.'s original task and showed that congenital learned helpless rats and acute pharmacological stress induced biases in their responding towards avoidance of punishment, further supporting a relationship between affective state and response bias in this task.

The aim of the present study was to use "reverse translation" to investigate whether a similar tone-based task could be used to study affective states in human participants. Unlike tasks that rely on inherently emotional cues such as facial images or words, this task requires emotional learning and memory, followed by the use of these emotional associations to make a judgement about a subsequent ambiguous cue in the same dimension. Previous studies have applied a principle similar to the one we investigate here, in which neutral stimuli are conditioned to emotional outcomes (Lissek et al., 2008; Lissek et al., 2010). Although their studies are more similar to the animal model of conditioned fear, Lissek and colleagues have validated a novel task for humans in which neutral cues (images of different-size rings) were paired with receiving either an electric shock $(\mathrm{CS}+)$ or no shock (CS-). When subsequently exposed to intermediate-size images, participants' responses revealed continuous gradients of conditioned fear generalization similar to those seen in animals (Lissek et al., 2008). Using a similar approach, electromyography recording revealed a much greater degree of generalisation in patients with panic disorder than in healthy controls (Lissek et al., 2010). In a task investigating the relationship between affective state and decision-making, Paul et al. (2011) used a computerbased task in which participants had to decide whether ambiguous and unambiguous predictor stimuli heralded resources or hazards. Similar to their findings in the rat cognitive affective bias task (Harding et al., 2004), they found that decision-making was slowed under both anxiety-like and depression-like states, as defined by scores on the Positive and Negative Affect Scale (PANAS).

\section{Method}

Materials and procedures

\section{Experiment 1: Task development}

Aversive stimulus comparison An opportunity sample of University of Bristol students ( $n=6,4$ female) were recruited and took part in an aversive stimulus comparison test. Two different audio stimuli known to be aversive were played to participants. The sound clips used were white noise and a baby crying (from www.thefreesoundproject. com). Participants were randomly played the two sound clips for 4, 12, and $25 \mathrm{~s}$. Aversion to the sound clips was recorded using visual analogue scales (VASs; 200-mm scale), ranging from not at all to the most ever, to assess overall aversion to the sound after each duration rating.

Pilot evaluation task The participants $(n=10,6$ female) completed the affective tone discrimination task (ATDT), with the baby crying (duration: $25 \mathrm{~s}$ ) as the aversive sound clip (as described below), without completing any questionnaire measures. Tone cues were presented for $5 \mathrm{~s}$. After testing, the participants were asked to complete three VASs (200-mm scale) to assess aversion to the sound clip of a baby crying (not at all to the most ever) and whether the degree of aversion changed across the duration of the study (no longer aversive to the most ever). For both pilot studies, participants received $£ 10$ at the end of the session.

\section{Experiment 2: ATDT study}

An opportunity sample of University of Bristol students were recruited via the Experimental Hours Scheme in the School of Experimental Psychology, and via advertisements 
around the University precinct $(n=40)$. The exclusion criteria comprised any history of migraine, diagnosed psychiatric illness, and antidepressant or anxiolytic drug treatment. Participants were asked to refrain from alcohol for at least $36 \mathrm{~h}$, and from caffeine for at least $6 \mathrm{~h}$, prior to testing. Each participant who completed the session was able to keep his or her winnings from the task, which was scored as the percentage accuracy to reward tones, with potential winnings of a maximum of $£ 10$. Those participants recruited via the Experimental Hours Scheme also received course credit. All participants were given detailed information about the procedures at least $24 \mathrm{~h}$ prior to the study, and they gave informed consent prior to participation. The study was approved by the Faculty of Science Research Ethics Committee.

Participants were randomly assigned to one of two conditions - reward tones associated with either the low or the high frequency - which were counterbalanced across participants. The dependent variables were the numbers of correct responses and the median correct response latencies for the eight tones (the two reference tones and six intermediate tones).

Experimental procedure A laptop computer (Toshiba Satellite Pro) was used to run the task, which was programmed in E-Prime Version 2.0 (Psychology Software Tools Inc., Sharpsburg, PA, USA) and presented on a 15.4-in. TFT monitor mounted immediately in front of the participant. Participants used a serial response box (Psychology Software Tools Inc.) to make their responses (a left or right keypress). A pair of $15-\mathrm{W}$ speakers was used to deliver the auditory tones, with sound clips set to a maximum volume of $80 \mathrm{~dB}$, which was measured at $1 \mathrm{~m}$ using a sound meter (Brüel \& Kjaer, Bremen, Germany). The auditory tones were produced using ToneGenerator Version 2.11 software (NCH Software, Australia, www.nch. com.au/tonegen/index.html) and the aversive sound clips obtained from www.thefreesoundproject.com.

Following consent procedures, participants sat in the experimentation room facing the monitor. All written instructions were presented on the monitor, which participants read in their own time. Understanding was confirmed by the investigator, with the opportunity for questions if necessary. The opening instructions gave examples of the tones and their outcomes. This was then followed by an explanation of the experimental procedure, and participants were informed that they would receive a monetary reward based on their accuracy to reward tones $(100 \%$ accuracy $=$ $£ 10.00$ ) and a punishment (a 25-s aversive sound clip of a baby crying) if they failed to respond correctly to avoidance tones.

Participants completed a training session with 16 trials (eight per reference tone, with frequencies of 500 or
$1000 \mathrm{~Hz}$ ) presented in a pseudorandom order. The tones were presented for $5 \mathrm{~s}$ and preceded by a central fixation cross for $0.5 \mathrm{~s}$. Participants had to select the correct left or right keypress response within this time or they would receive a 1.5 -s timeout, with the words "Too Slow" presented on screen. Correct responses to the reward tone were followed by an image of money, while correct responses to the avoidance tone were followed by an image representing successful avoidance of the aversive sound clip. Incorrect responses to the reward tone were followed by a 1.5 -s timeout, while incorrect responses to the avoidance tone were followed by a 25 -s aversive sound clip. The intertrial interval was $2 \mathrm{~s}$. All participants were required to achieve $100 \%$ accuracy for both the reward and avoidance tones before progressing to the testing phase of the task.

After a brief pause during which they could ask questions, participants started the testing session, which consisted of the reference tones $(500$ and $1000 \mathrm{~Hz}, 15$ trials per tone) and ambiguous probe tones (six tones at $20-\mathrm{Hz}$ intervals on either side of the $750-\mathrm{Hz}$ midpoint; 15 trials per intermediate tone, for a total of 90 trials), presented in pseudorandom order. The ambiguous tones were counterbalanced with the reference tones and were termed A1-A3 (A3 being the most ambiguous avoidance tone) and R1-R3 (R3 being the most ambiguous reward tone). All other conditions were the same as in the training session. The outcomes for the ambiguous tones were the same as for the reference tone that they were more closely associated with. At the end of the task, participants completed questionnaire and VAS measures and were then given the cash equivalent of the reward accrued during the task, debriefed, and given an opportunity to ask questions. The questionnaire measures included the Beck Depression Inventory (BDI; Beck, Ward, Mendelson, Mock, \& Erbaugh, 1961), the State-Trait Anxiety Inventory (STAI) State and Trait subscales (Spielberger, 1983), the Patient Health Questionnaire-9 (PHQ-9; Kroenke, Spitzer, \& Williams, 2001), the Positive and Negative Affect Schedule (PANAS; Watson, Clark, \& Tellegen, 1988), and the Behavioral Inhibition/Behavioral Activation Scales (BIS/BAS; Carver \& White, 1994). The participants also completed 200-mm VASs for depression, anxiety, optimism, and pessimism to determine subjective ratings of mood.

Statistical analysis

\section{Experiment 1: Task development}

Aversive stimulus comparison Measurements given on the VAS scales were analysed using repeated measures ANOVA with Duration of the Sound Clip (4, 12, or 25 s) and Sound Clip Type (white noise or baby crying) as within-subjects 
factors. Pairwise least significant difference comparisons were also performed post hoc.

Pilot evaluation task The numbers of correct responses and the median latency correct reaction time (RT) data were recorded for the pilot study and analysed using a repeated measures ANOVA, with Ambiguity $(60,40$, or $20 \mathrm{~Hz}$ ) and Valence (reward or avoid) as within-subjects factors. For the RT analyses, the data from trials with error responses were removed and not analysed further, and analyses were conducted on interparticipant means of the median RTs using repeated measures ANOVA as described above.

\section{Experiment 2: ATDT study}

Response selection and the latency correct RT data were recorded and analysed using a repeated measures ANOVA, with Ambiguity $(60,40$, or $20 \mathrm{~Hz}$ ) and Valence (reward or avoid) as within-subjects factors. For the RT analyses, the data from trials with error responses were removed $(18 \%$ of the data) and not analysed further, and analyses were conducted on interparticipant means of the median RTs. Bias scores were calculated by subtracting the numbers of responses or RTs to obtain reward from the numbers of responses or RTs to avoid punishment at maximum ambiguity. These bias scores generated single values reflecting whether the participant made more responses to obtain reward versus to avoid punishment or were quicker to respond for reward- versus punishment-related cues during probe trials at maximum ambiguity. This value was used for correlation analyses with outcome measures from the questionnaire and VAS data. Age was included as a covariate in all analyses. A Greenhouse-Geisser correction was used to adjust for violation of the sphericity assumption, and an alpha level of .05 was retained throughout.

\section{Results}

Experiment 1: Task development

Aversive stimulus comparison Repeated measures ANOVAs indicated significant main effects of duration $[F(1.14,5.68)=$ $15.57, p=.008]$ and sound clip $[F(1.00,5.00)=32.26, p=$ $.002]$, although no interaction was found $[F(1.10,5.51)=$ $1.41, p=.290]$. Pairwise least-significant-difference comparisons between the sound clips revealed that the baby crying was significantly more aversive than white noise for all durations, with the longest duration of baby crying being the most aversive (data not shown).

Pilot evaluation task The numbers of correct responses were plotted for each degree of ambiguity for both valences
(60, 40, and $20 \mathrm{~Hz}$ on either side of the midpoint; see Fig. 1), with a highly significant main effect of ambiguity being observed $[F(1.23,14.02)=25.41, p<.001]$. No effect of valence or interaction was found, $p>.2$.

Marginal main effects of ambiguity and valence were found for median latencies correct $[F(1.18,10.66)=3.81$, $p=.073$, and $F(1.00,9.00)=3.75, p=.085$, respectively; see Fig. 1], suggesting that the latency to correctly respond to cues was dependent on the difficulty to identify the tone and the associated valence outcome, although no interaction effect was observed, $p>.5$. The VAS scores (see Fig. 1 bottom) to the aversiveness of the sound clip of a baby crying given by participants in the pilot study were comparable to those in the aversive stimulus study, and ratings were strongly affected by the duration of the sound clip, with very little habituation being reported and responses being around the halfway mark of the scale, suggesting that aversiveness was unchanged.

\section{Experiment 2: ATDT study}

The data from 2 participants were excluded from all analyses due to low accuracy of responding to the reference tones $(<90 \%)$, resulting in a final sample of $n=38$ for analysis. The participants (55\% female) were 18-48 years old (average 25 years, $S D=8$ ).

Response choice Participants achieved greater than $90 \%$ accuracy for the reference tones, and no significant differences between the reference tones for reward and punishment were observed. A repeated measures ANOVA of the response choice data indicated a significant main effect of ambiguity $[F(1.90,68.46)=6.80, p=.002]$, indicating that accuracy was reduced relative to the degree of tone ambiguity. A marginal main effect of tone valence $[F(1.00$, $36.00)=4.01, p=.053]$ was also observed, which was qualified by a significant Ambiguity $\times$ Valence interaction $[F(1.83,65.85)=3.41, p=.043]$. This effect was due largely to a bias towards avoiding punishment at the greatest level of ambiguity (i.e., $20 \mathrm{~Hz}$ from the midpoint), although a post hoc paired-samples $t$ test showed this bias not to be significant $(p=.141)$. These data are presented in Fig. 2 .

Response latencies A repeated measures ANOVA of the RT data indicated a significant main effect of ambiguity $[F$ $(1.55,55.92)=6.39, p=.006]$, reflecting slower RTs with increasing ambiguity. No other main effects or interactions were significant $(p s>.12)$. These data are presented in Fig. 3. The marginal main effect of valence seen in the pilot experiment was not replicated in this main study.

Correlation analyses In order to facilitate correlation analyses, a bias score was derived by subtracting the number and 

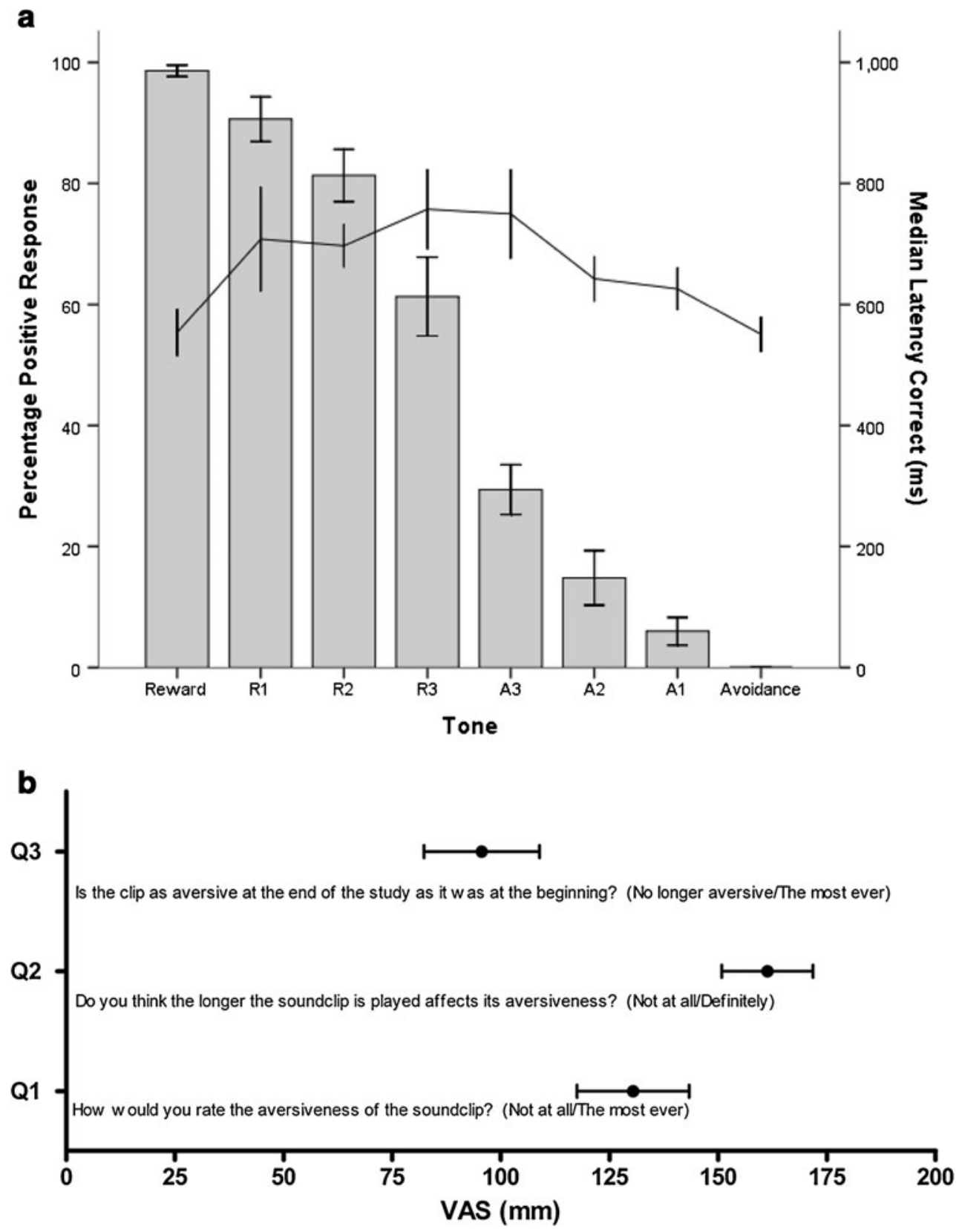

Fig. 1 (Top) Results from a pilot study to investigate the task variables and aversive stimuli associated with the human affective tone discrimination task: Participants made fewer responses to obtain reward relative to the distance of the tone from the reference frequency (bars), with a significant main effect of ambiguity $[F(1.56,14.02)=25.41, p<$ $.001]$. These data are consistent with the results from rodent studies. Analysis of the response latency data (line) revealed a tendency towards a slowing of reaction times that reflected the degree of ambiguity of the probe tone $[F(1.18,10.66)=3.809, p=.073]$. In this pilot study, there was no significant Ambiguity $\times$ Valence interaction $[F(1.69$,

RT of responses to obtain reward from the number and RT of responses to avoid punishment at maximum ambiguity (see Figs. 2 and 3). A positive score, therefore, reflected increased responding to avoid punishment relative to obtaining reward and a slowing of responses to avoidance relative
$15.24)=0.53, p=.568$, although the small number of participants $(n=10)$ meant that this study lacked statistical power. The results are shown as means $\pm S E M$ s. Reward = reference tone for obtaining reward, Avoidance $=$ reference tone for avoidance of punishment. Among the ambiguous reward tones (R1-R3) and ambiguous avoidance tones (A1-A3), R3 and $\mathrm{A} 3$ represent the most ambiguous tones ( $20 \mathrm{~Hz}$ from the midpoint). (Bottom) After completion of the pilot study, participants completed three visual analogue scales rating the aversiveness of the sound clip. Data are shown as means $\pm S E M \mathrm{~s}$

to reward. The choice bias score showed a significant positive correlation with VAS anxiety $(r=+.33, p=.045)$ and a trend-level positive correlation with STAI state anxiety $(r=+.27, p=.099)$. Controlling for depressed mood as measured by the BDI and PHQ-9 strengthened these 


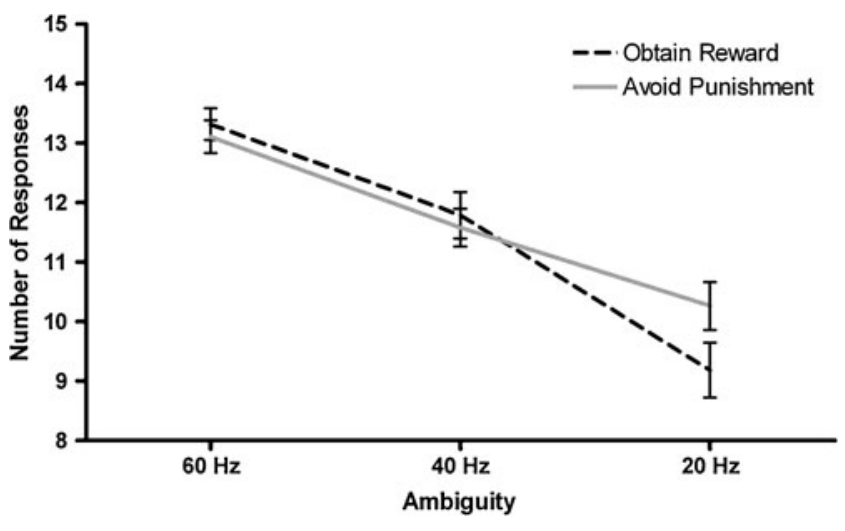

Fig. 2 Correct responses made to probe tones in the ATDT study: Results for correct responses made to obtain reward or avoid punishment during the presentation of ambiguous cues. A total of six ambiguous cues (probe tones) were used, played at frequencies $20 \mathrm{~Hz}$ successively farther on either side from the midpoint. The numbers of responses to obtain reward and avoid punishment are presented, plotted against increasing levels of tone ambiguity relative to the midpoint and their associated valences. A repeated measures ANOVA of the response choice data indicated a significant main effect of ambiguity $[F(1.90,68.46)=6.80, p=.002]$ and a marginal main effect of valence $[F(1,36)=4.01, p=.053]$, which were qualified by a significant Ambiguity $\times$ Valence interaction $[F(1.83,65.85)=3.41, p=$ $.043]$. The data are shown as means $\pm S E M \mathrm{~s}, n=38$

correlations for both VAS anxiety $(r=+.35, p=.036)$ and STAI state anxiety $(r=+.35, p=.035)$. No other correlations were significant $(p s>.13)$. These data are presented in Table 1.

The RT bias score did significantly correlate with some motivational measures, but no significant correlation to anxiety or affective state was seen, even when controlling for depressed mood. These data are presented in Table 2.

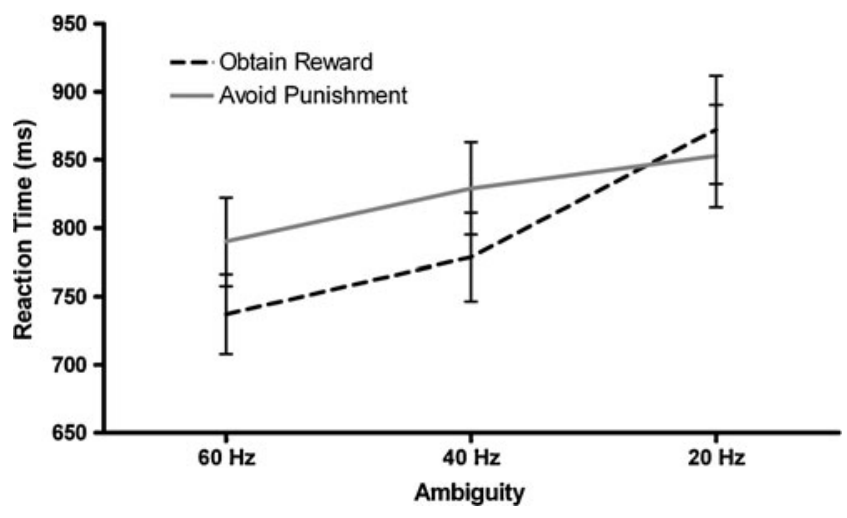

Fig. 3 Reaction times (RTs) for correct probe tone responses in the ATDT study: Responses correctly made to ambiguous cues to obtain reward and avoid punishment. Mean response latencies are presented, plotted against increasing levels of tone ambiguity relative to the midpoint and their associated valences. A total of six ambiguous cues (probe tones) were used, played at frequencies $20 \mathrm{~Hz}$ successively farther on either side from the midpoint. A repeated measures ANOVA of the RT data indicated a significant main effect of ambiguity $[F(1.55$, $55.92)=6.39, p=.006]$, but no significant effect of valence was observed. The data are shown as means \pm SEMs, $n=38$

\section{Discussion}

These data demonstrate the direct translation of a rodent model of affective behaviour into a human task and suggest that this task is sensitive to affective biases in humans. In the pilot study, we obtained data for response choices and RTs that closely mirror the findings in normal rats (Enkel et al., 2010; Harding et al., 2004). The baby crying sound clip was aversive to all participants when compared to white noise, and this degree of aversion increased relative to the duration of the clip. Participants rated the baby crying as similarly aversive at the start and end of the task, suggesting that they did not habituate over the duration of the task. The results from the main experiment suggest that this methodology provides a measure of state anxiety in humans. The choice bias score calculated for each participant revealed an overall bias towards avoiding punishment, and the degree of choice bias was found to correlate to self-report and state anxiety measures, which were strengthened when controlling for depressed mood. Due to the multiple correlations used in this analysis, speculation on how the results of choice relate to anxiety measures must be taken with caution.

In this type of task, both rats (Enkel et al., 2010; Harding et al., 2004) and humans tend to show a bias towards avoidance of punishment. It is not clear whether this is due to the relative values of reward versus avoidance outcomes, but it suggests that under the given task conditions, both species tend to choose to avoid punishment at the highest level of ambiguity. Unlike the studies in rats (Enkel et al., 2010; Harding et al., 2004), in our study the degree of ambiguity was not related to the degree of bias observed, and only the midpoint data were significant. The rat work had used animals in putative negative affective states induced by pharmacological or ethological manipulations. In this study, we report findings from a healthy human population, and further studies in patients will be necessary before we can conclude whether a range of ambiguous cues is necessary or whether a single midpoint cue would suffice (Paul et al., 2011). In previous studies, Lissek and colleagues found that the degree of ambiguity of the cue did relate to response bias and that this effect was influenced by anxiety-related pathology (Lissek et al., 2008; Lissek et al., 2010).

The significant correlation between self-report measures of anxiety and choice bias score suggest that the task is sensitive to individual differences in baseline anxiety, where higher levels of self-reported anxiety correlate with increased avoidance of punishment. The findings are similar to those of previous studies that have shown negative affective biases in clinically anxious patients and control participants with high trait or induced state anxiety (Mathews \& MacLeod, 1994; Richards et al., 2002). Whilst a clear correlation between bias score and anxiety state was observed 
Table 1 Results from correlation analyses between the response bias score in the ATDT and questionnaire measures

${ }^{\dagger}$ Trend correlation, becomes significant when depression controlled. *Significant correlations. BDI, Beck Depression Inventory; PHQ-9, Patient Health Questionnaire-9; BAS, Behavioral Activation Scale; BIS, Behavioral Inhibition Scale; PANAS, Positive and Negative Affect Schedule; STAI, StateTrait Anxiety Inventory.

\begin{tabular}{lllll}
\hline & Bivariate Correlation & $p$ Value & Partial Correlation & $p$ Value \\
\hline BDI & +.06 & .73 & $\mathrm{n} / \mathrm{a}$ & \\
PHQ-9 & +.15 & .36 & $\mathrm{n} / \mathrm{a}$ & .72 \\
BAS Drive & -.01 & .96 & +.06 & .43 \\
BAS Fun Seeking & +.15 & .37 & +.14 & .78 \\
BAS Reward & -.07 & .67 & -.05 & .26 \\
BIS & +.20 & .23 & +.19 & .74 \\
PANAS Positive & -.10 & .54 & -.06 & .10 \\
PANAS Negative & +.25 & .14 & +.28 & $.035^{*}$ \\
STAI State & +.27 & $.099^{\dagger}$ & +.35 & .48 \\
STAI Trait & +.16 & .35 & +.12 & .38 \\
VAS Depression & +.16 & .33 & +.15 & $.036^{*}$ \\
VAS Anxiety & +.33 & $.045^{*}$ & +.35 & .44 \\
VAS Pessimism & -.15 & .38 & -.13 &
\end{tabular}

in this task, RT bias scores were not correlated with anxiety. The data obtained in this study show that participants have increased RTs when responding to the more ambiguous tones, but that this effect is not sensitive to valence or related to individual differences in affective state. In contrast to the anxiety measures, questionnaire data relating to drive were correlated with RT bias scores. As this measure did not correlate with PANAS or anxiety scores, these individual differences in latencies to respond to obtain reward versus avoid punishment are more likely due to motivational differences.

In the rat versions of this task, effects on RTs following chronic mild stress (Harding et al., 2004) or a pharmacological stress challenge (Enkel et al., 2010) were observed, with animals showing faster RTs to avoidance of punishment. It is not clear whether the lack of a main effect of valence on RTs in the human version of this task is due to the population used (healthy volunteers vs. a clinical population) or is a limitation of the task. In other paradigms, a relationship between RTs, emotional valence, and anxiety has been seen, although mainly in clinical populations (Bar-Haim, Lamy, Pergamin, Bakermans-Kranenburg, \& van Ijzendoorn, 2007; Calvo \& Eysenck, 2008; Ferguson, Moghaddam, \& Bibby, 2007; Miu, Heilman, \& Houser, 2008). The fact that measures of anxiety but not depression were found to correlate to task performance suggests that the method we used is more sensitive to decision-making deficits associated with anxiety than to the negative judgement and memory biases observed in depression (for reviews, see Bar-Haim et al., 2005; Harmer et al., 2004; Mathews \& MacLeod, 2005; Mogg \& Bradley, 2002; Wright \& Bower, 1992).

This study focused on investigating the affective tone discrimination task in a healthy population with normal variations in mood state, rather than in a clinical population. In our tested population, the results suggest that anxiety measures are related to performance differences in the task,
Table 2 Results from correlation analyses between the reaction time bias score in the ATDT and questionnaire measures

*Significant correlations. BDI, Beck Depression Inventory; PHQ-9, Patient Health Questionnaire 9; BAS, Behavioral Activation Scale; BIS, Behavioral Inhibition Scale; PANAS, Positive and Negative Affect Schedule; STAI, State-Trait Anxiety Inventory

\begin{tabular}{lllll}
\hline & Bivariate Correlation & $p$ Value & Partial Correlation & $p$ Value \\
\hline BDI & .044 & .795 & $\mathrm{n} / \mathrm{a}$ & \\
PHQ-9 & -.018 & .914 & $\mathrm{n} / \mathrm{a}$ & $.004^{*}$ \\
BAS Drive & -.421 & $.008^{*}$ & -.469 & $.003^{*}$ \\
BAS Fun Seeking & -.481 & $.002^{*}$ & -.477 & .080 \\
BAS Reward & -.281 & .087 & -.296 & $.008^{*}$ \\
BIS & .345 & $.034^{*}$ & .437 & .784 \\
PANAS Positive & .021 & .901 & .047 & .879 \\
PANAS Negative & .007 & .966 & -.026 & .511 \\
STAI State & .121 & .469 & .113 & .078 \\
STAI Trait & .190 & .254 & .298 & .912 \\
VAS Depression & .005 & .978 & -.019 & .953 \\
VAS Anxiety & .013 & .938 & -.010 & .299 \\
VAS Pessimism & -.177 & .288 & -.178 & \\
\hline
\end{tabular}


although replication of these findings will be necessary before it can be clear that these two measures are related. It would also be useful to undertake further studies in clinical populations to determine how choice bias and RTs differ with disease state and severity. These experiments will also be necessary to provide a more direct comparison with the rodent literature, where chronic mild stress (Harding et al., 2004), congenital learned helplessness, and acute pharmacological challenges (Enkel et al., 2010) have been used to induce a change in baseline affective state. A lack of animal assays suitable for quantifying the emotional impairments that are core to diseases such as anxiety and depression has been seen as a major limitation in the development of new therapeutic interventions. We have shown that it is possible to "reverse translate" an animal paradigm and to develop an equivalent task for human participants, with the results obtained suggesting an association between task performance and state anxiety.

Author note We kindly thank all participants for their willingness to contribute to this study and the members within the lab for their proofreading skills. We also thank Angela Attwood and the rest of the Tobacco and Alcohol Research Group (TARG) group for their mentoring of M.H.A. This research was supported by a Biotechnology and Biological Sciences Research Council (BBSRC) PhD studentships awarded to M.H.A. and C.H. The BBSRC had no further role in the study design; in the collection, analysis, and interpretation of the data; in the writing of the report; and in the decision to submit the article for publication. E.S.J.R. was funded by a Research Councils U.K. Academic Fellowship supported by the British Pharmacological Society Integrative Pharmacology Fund. The authors have no relevant conflicts of interest to declare.

\section{References}

Bar-Haim, Y., Lamy, D., \& Glickman, S. (2005). Attentional bias in anxiety: A behavioral and ERP study. Brain and Cognition, 59, $11-22$.

Bar-Haim, Y., Lamy, D., Pergamin, L., Bakermans-Kranenburg, M. J., \& van Ijzendoorn, M. H. (2007). Threat-related attentional bias in anxious and nonanxious individuals: A meta-analytic study. Psychological Bulletin, 133, 1-24.

Beck, A. T., Ward, C. H., Mendelson, M., Mock, J., \& Erbaugh, J. (1961). An inventory for measuring depression. Archives of General Psychiatry, 4, 561-571.

Bradley, B. P., Mogg, K., \& Millar, N. (1996). Implicit memory bias in clinical and non-clinical depression. Behaviour Research and Therapy, 34, 865-879.

Brilot, B. O., Asher, L., \& Bateson, M. (2010). Stereotyping starlings are more "pessimistic. Animal Cognition, 13, 721-731.

Brydges, N. M., Leach, M., Nicol, K., Wright, R., \& Bateson, M. (2011). Environmental enrichment induces optimistic cognitive bias in rats. Animal Behaviour, 81, 169-175.

Calvo, M. G., \& Eysenck, M. W. (2008). Affective significance enhances covert attention: Roles of anxiety and word familiarity. Quarterly Journal of Experimental Psychology, 61, 1669-1686. doi:10.1080/17470210701743700

Carver, C. S., \& White, T. L. (1994). Behavioral inhibition, behavioral activation, and affective responses to impending reward and punishment: The BIS/BAS scales. Journal of Personality and Social Psychology, 67, 319-333. doi:10.1037/00223514.67.2.319

Chamberlain, S. R., \& Sahakian, B. J. (2006). The neuropsychology of mood disorders. Current Psychiatry Reports, 8, 458-463.

Doyle, R. E., Lee, C., Deiss, V., Fisher, A. D., Hinch, G. N., \& Boissy, A. (2011). Measuring judgement bias and emotional reactivity in sheep following long-term exposure to unpredictable and aversive events. Physiology \& Behavior, 102, 503-510.

Elliott, R., Rubinsztein, J. S., Sahakian, B. J., \& Dolan, R. J. (2002). The neural basis of mood-congruent processing biases in depression. Archives of General Psychiatry, 59, 597-604.

Elliott, R., Zahn, R., Deakin, J. F. W., \& Anderson, I. M. (2011). Affective cognition and its disruption in mood disorders. Neuropsychopharmacology, 36, 153-182.

Enkel, T., Gholizadeh, D., von Bohlen und Halbach, O., SanchisSegura, C., Hurlemann, R., Spanagel, R., . . . Vollmayr, B. (2010). Ambiguous-cue interpretation is biased under stress- and depression-like states in rats. Neuropsychopharmacology, 35, 1008-1015. doi:10.1038/npp.2009.204

Erickson, K., Drevets, W. C., Clark, L., Cannon, D. M., Bain, E. E., Zarate, C. A., Jr., . . Sahakian, B. J. (2005). Mood-congruent bias in affective go/no-go performance of unmedicated patients with major depressive disorder. American Journal of Psychiatry, 162, 2171-2173. doi:10.1176/appi.ajp.162.11.2171

Ferguson, E., Moghaddam, N. G., \& Bibby, P. A. (2007). Memory bias in health anxiety is related to the emotional valence of healthrelated words. Journal of Psychosomatic Research, 62, 263-274.

Frazer, A., \& Morilak, D. A. (2005). What should animal models of depression model? Neuroscience \& Biobehavioral Reviews, 29, $515-523$.

Gotlib, I. H., Ranganath, C., \& Rosenfeld, J. P. (1998). Frontal EEG alpha asymmetry, depression, and cognitive functioning. Cognition and Emotion, 12, 449-478.

Harding, E. J., Paul, E. S., \& Mendl, M. (2004). Animal behaviour: Cognitive bias and affective state. Nature, 427, 312-312.

Harmer, C. J., Shelley, N. C., Cowen, P. J., \& Goodwin, G. M. (2004). Increased positive versus negative affective perception and memory in healthy volunteers following selective serotonin and norepinephrine reuptake inhibition. American Journal of Psychiatry, 161, 1256-1263. doi:10.1176/appi.ajp.161.7.1256

Kroenke, K., Spitzer, R. L., \& Williams, J. B. (2001). The PHQ-9: Validity of a brief depression severity measure. Journal of General Internal Medicine, 16, 606-613.

LeDoux, J.E. (1995). Emotional processing, but not emotions, can occur unconsciously The Nature of Emotion: Fundamental Questions (pp. 291-292): OUP USA

LeDoux, J. (1999). The Emotional Brain: The Mysterious Underpinnings of Emotional Life: Phoenix.

Leppänen, J. M. (2006). Emotional information processing in mood disorders: A review of behavioral and neuroimaging findings. Current Opinion in Psychiatry, 19, 34-39. doi:10.1097/01. yco.0000191500.46411.00

Leppänen, J. M., Milders, M., Bell, J. S., Terriere, E., \& Hietanen, J. K. (2004). Depression biases the recognition of emotionally neutral faces. Psychiatry Research, 128, 123-133.

Lissek, S., Biggs, A. L., Rabin, S. J., Cornwell, B. R., Alvarez, R. P., Pine, D. S., et al. (2008). Generalization of conditioned fearpotentiated startle in humans: experimental validation and clinical relevance. Behav Res There, 46(5), 678-687.

Lissek, S., Rabin, S., Heller, R. E., Lukenbaugh, D., Geraci, M., Pine, D. S., et al. (2010). Overgeneralization of conditioned fear as a pathogenic marker of panic disorder. Am J Psychiatry, 167(1), 47-55.

Mathews, A., \& MacLeod, C. (1994). Cognitive approaches to emotion and emotional disorders. Annual Review of Psychology, 45, 25-50. doi:10.1146/annurev.ps.45.020194.000325 
Mathews, A., \& MacLeod, C. (2005). Cognitive vulnerability to emotional disorders. Annual Review of Clinical Psychology, 1, 167195. doi:10.1146/annurev.clinpsy.1.102803.143916

McArthur, R., \& Borsini, F. (2006). Animal models of depression in drug discovery: A historical perspective. Pharmacology Biochemistry and Behavior, 84, 436-452.

Mitterschiffthaler, M. T., Williams, S. C. R., Walsh, N. D., Cleare, A. J., Donaldson, C., Scott, J., \& Fu, C. H. Y. (2008). Neural basis of the emotional Stroop interference effect in major depression. Psychological Medicine, 38, 247-256. doi:10.1017/S0033291707001523

Miu, A. C., Heilman, R. M., \& Houser, D. (2008). Anxiety impairs decision-making: Psychophysiological evidence from an Iowa Gambling Task. Biological Psychology, 77, 353-358.

Mogg, K., \& Bradley, B. P. (2002). Selective orienting of attention to masked threat faces in social anxiety. Behaviour Research and Therapy, 40, 1403-1414. doi:10.1016/S0005-7967(02)00017-7

Mogg, K., Bradley, B. P., Millar, N., \& White, J. (1995). A follow-up study of cognitive bias in generalized anxiety disorder. Behaviour Research and Therapy, 33, 927-935.

Mogg, K., Bradley, B. P., Williams, R., \& Mathews, A. (1993). Subliminal processing of emotional information in anxiety and depression. Journal of Abnormal Psychology, 102, 304-311.

Murphy, F. C., Sahakian, B. J., Rubinsztein, J. S., Michael, A., Rogers, R. D., Robbins, T. W., \& Paykel, E. S. (1999). Emotional bias and inhibitory control processes in mania and depression. Psychological Medicine, 29, 1307-1321. doi:10.1017/S0033291799001233
Paul, E. S., Harding, E. J., \& Mendl, M. (2005). Measuring emotional processes in animals: The utility of a cognitive approach. Neuroscience and Biobehavioral Reviews, 29, 469-491.

Paul, E. S., Cuthill, I., Kuroso, G., Norton, V., Woodgate, J., \& Mendl, M. (2011). Mood and the speed of decisions about anticipated resources and hazards. Evolution and Human Behavior, 32, 2128. doi:10.1016/j.evolhumbehav.2010.07.005

Richards, A., French, C. C., Calder, A. J., Webb, B., Fox, R., \& Young, A. W. (2002). Anxiety-related bias in the classification of emotionally ambiguous facial expressions. Emotion, 2, 273-287.

Salmeto, A. L., Hymel, K. A., Carpenter, E. C., Brilot, B. O., Bateson, M., \& Sufka, K. J. (2011). Cognitive bias in the chick anxietydepression model. Brain Research, 1373, 124-130.

Spielberger, C. D. (1983). Manual for the State-Trait Anxiety Inventory. Palo Alto, CA: Consulting Psychologists Press.

Torres, C., Cándido, A., Escarabajal, M. D., de la Torre, L., Maldonado, A., Tobeña, A., \& Fernández-Teruel, A. (2005). Successive negative contrast in one-way avoidance learning in female roman rats. Physiology and Behavior, 85, 377-382. doi:10.1016/j.physbeh.2005.02.023

Watson, D., Clark, L. A., \& Tellegen, A. (1988). Development and validation of brief measures of positive and negative affect: The PANAS scales. Journal of Personality and Social Psychology, 54, 1063-1070.

Wright, W. F., \& Bower, G. H. (1992). Mood effects on subjective probability assessment. Organizational Behavior and Human Decision Processes, 52, 276-291. doi:10.1016/0749-5978(92)90039-A 